\title{
CHARACTERISTIC ROOTS OF $M$-MATRICES
}

\section{DOUGLAS E. CRABTREE}

A square matrix $B$ is called a nonnegative matrix (written $B \geqq 0$ ) if each element of $B$ is a nonnegative number. It is well known [1] that every nonnegative matrix $B$ has a nonnegative characteristic root $p(B)$ (the Perron root of $B$ ) such that each characteristic root $\lambda$ of $B$ satisfies $|\lambda| \leqq p(B)$.

A square matrix $A$ is called an $M$-matrix if it has the form $k \cdot I-B$, where $B$ is a nonnegative matrix, $k>p(B)$, and $I$ denotes the identity matrix. In case $A$ is a real, square matrix with nonpositive off-diagonal elements, each of the following is a necessary and sufficient condition for $A$ to be an $M$-matrix [4, p. 387].

(1) Each principal minor of $A$ is positive.

(2) $A$ is nonsingular, and $A^{-1} \geqq 0$.

(3) Each real characteristic root of $A$ is positive.

(4) There is a row vector $x$ with positive entries $(x>0)$ such that $x A>0$.

If $A$ is an $M$-matrix, then $A$ has a positive characteristic root $q(A)$ which is minimal, in the sense that for each characteristic $\operatorname{root} \beta$ of $A$, $q(A) \leqq|\beta|[4$, p. 389]. Bounds for $q(A)$ can be readily obtained using known bounds for the Perron root of a nonnegative matrix. In this paper, as in [2], we reverse this procedure, studying the characteristic roots of $M$-matrices in order to find new bounds for Perron roots.

Ky Fan proved the following lemma [3]:

Lemma A. Let $A=\left(a_{i j}\right)$ be an $M$-matrix of order $n$. Then the matrix $C=\left(c_{i j}\right)$ given by

$$
c_{i j}=a_{i j}-a_{i n} a_{n j}\left(1 / a_{n n}\right) \quad(i, j=1,2, \cdots, n-1) .
$$

is an $M$-matrix, and $c_{i j} \leqq a_{i j}(i, j=1,2, \cdots, n-1)$.

In [2] we generalized Lemma $A$ by using certain principal minors in place of single elements of $A$. Here we prove a different generalization of Fan's lemma, contained in Theorem 1.

We denote the submatrix of a matrix $A$ formed using rows $i_{1}, i_{2}, \cdots, i_{p}$ and columns $j_{1}, j_{2}, \cdots j_{p}$ by $A\left(i_{1}, i_{2}, \cdots, i_{p}\right.$; $\left.j_{1}, j_{2}, \cdots, j_{p}\right)$. For principal submatrices we abbreviate this to $A\left(i_{1}, i_{2}, \cdots, i_{p}\right) . A_{i, j}$ denotes the element of $A$ in row $i$ and column $j$.

Presented to the Society, January 24, 1966; received by the editors December 13, 1965. 
THEOREM 1. Let $M$ be an $M$-matrix of order $n=m k$, partitioned into the form

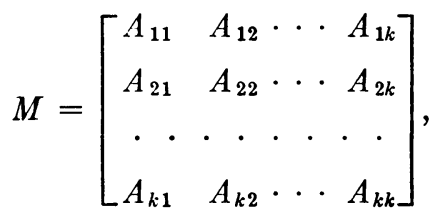

where each $A_{i j}$ is an $m \times m$ matrix. Let $\phi(M)$ denote the matrix

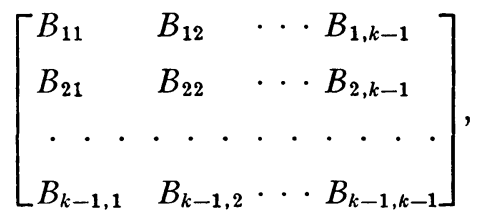

where $B_{i j}=A_{i j}-A_{i k} A_{\mathbf{k}}^{-1} A_{k j}$. Then

(5) $\phi(M)$ is an $M$-matrix, of order $m(k-1)$,

(6) $\phi(M)_{i, j} \leqq M_{i, j}$, for $i, j=1,2, \cdots, m(k-1)$,

(7) $\operatorname{det} \phi(M)=\operatorname{det} M / \operatorname{det} A_{k k}$, and

(8) $q(\phi(M)) \geqq q(M)$.

The proof of the theorem depends on two lemmas.

LeMma 1. Let $T=\left(t_{i j}\right)$ be an $M$-matrix of order $n$, with minimal characteristic root $q(T)$. Let $\lambda$ be a number such that $\lambda<q(T)$. Then $\operatorname{det}(T-\lambda I)>0$.

Proof. If $\omega_{1}, \omega_{2}, \cdots, \omega_{n}$ are the characteristic roots of $T, \operatorname{det}(T-\lambda I)$ $=\prod_{i=1}^{n}\left(\omega_{i}-\lambda\right)$. For each real $\omega_{i}, \omega_{i} \geqq q(T)>\lambda$, so that $\omega_{i}-\lambda>0$. The complex factors $\omega_{i}-\lambda$ occur in conjugate pairs whose product is positive. Thus $\operatorname{det}(T-\lambda I)>0$.

LeMma 2. Let $S=\left(s_{i j}\right)$ be an $M$-matrix of order $n$, let $\lambda$ be a number such that $0<\lambda<q(S)$, and let $t$ be an integer such that $0 \leqq t<n$. Then the determinant of the matrix obtained from $S$ by subtracting $\lambda$ from each of the first $t$ main diagonal elements $s_{11}, s_{22}, \cdots, s_{t t}$ is positive.

Proof. Let $T$ be the matrix obtained from $S$ by adding $\lambda$ to the main diagonal elements $s_{t+1, t+1}, \cdots, s_{n, n}$. Since $\lambda>0, T \geqq S$ and the off-diagonal elements of $T$ are nonpositive, so [4, p. 389] $T$ is an $M$ matrix and $q(T) \geqq q(S)$. Thus, since $\lambda<q(T)$, Lemma 1 implies that $\operatorname{det}(T-\lambda I)>0$, which proves Lemma 2 .

Proof of Theorem 1 . Since $M$ has nonpositive off-diagonal elements, $A_{i k}$ and $A_{k j}$ are nonpositive matrices if $i, j \neq k$. Also $A_{k k}$, a principal submatrix of $M$, is itself an $M$-matrix [4, p. 390], so that 
$A_{k k}^{-1} \geqq 0$. Thus $A_{i k} A_{k k}^{-1} A_{k j} \geqq 0$, so that $B_{i j} \leqq A_{i j}$, verifying (6). So $\phi(M)$ has nonpositive off-diagonal elements, and to show that $\phi(M)$ is an $M$-matrix we prove now that each principal minor of $\phi(M)$ is positive.

Let $F$ be the nonnegative, $n \times n$ matrix

$$
F=\left[\begin{array}{cc}
I & 0 \\
0 & A_{k k}^{-1}
\end{array}\right] \text {. }
$$

Then

$$
M F=\left[\begin{array}{ccc}
A_{11} \cdots & A_{1 k} A_{k k}^{-1} \\
A_{21} \cdots & A_{2 k} A_{k k}^{-1} \\
\cdot & \cdot & \cdot \\
A_{k 1} & \cdots & I
\end{array}\right]
$$

has nonpositive off-diagonal elements. Also, by (4), since $M$ is an $M$-matrix, there is a vector $x$ with positive entries $(x>0)$ such that $x M>0$. Then also $(x M) F>0$, since $F \geqq 0$ and $F$ has at least one positive element in each column. (Each main diagonal element of the inverse of an $M$-matrix, being the quotient of two positive principal minors, is positive.) Thus, by (4), $M F$ is an $M$-matrix. Moreover, $\phi(M)=\phi(M F)$, so it suffices to show that each principal minor of $\phi(M F)$ is positive.

To this end, write

$$
M F=\left[\begin{array}{cccc}
C_{11} & C_{12} & \cdots & C_{1 k} \\
C_{21} & C_{22} & \cdots & C_{2 k} \\
\cdot & \cdot & \cdot & \cdot \\
C_{k 1} & C_{k 2} & \cdots & \cdot
\end{array}\right],
$$

with $C_{i j}=A_{i j}$ if $j \neq k$, and $C_{i k}=A_{i k} A_{k k}^{-1}$. Then it is easily verified that a typical element of $\phi(M F)$, say $\left(C_{i j}-C_{i k} C_{k j}\right)_{\alpha, \beta}$, can be written as the determinant

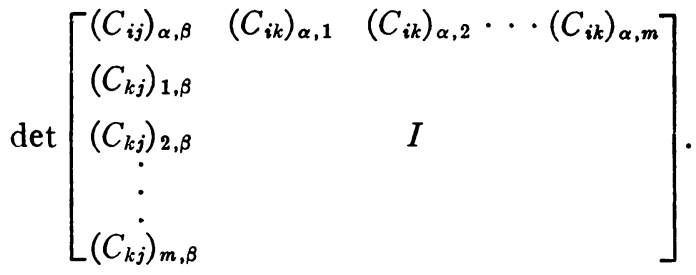

Thus by Sylvester's identity [5, p. 16], if $\phi(M F)\left(i_{1}, i_{2}, \cdots, i_{t}\right)$ is a principal submatrix of $\phi(M F)$, then 
$\operatorname{det}\left\{\phi(M F)\left(i_{1}, i_{2}, \cdots, i_{t}\right)\right\}$

$$
=(\operatorname{det} I)^{t-1} \operatorname{det}(M F)\left(i_{1}, i_{2}, \cdots, i_{t}, n-m+1, \cdots, n\right)>0 .
$$

This completes the proof that $\phi(M)$ is an $M$-matrix.

To verify (7), we use Sylvester's identity again to write

$$
\begin{aligned}
\operatorname{det}(\phi(M)) & =\operatorname{det} \phi(M F)=\operatorname{det} M F=\operatorname{det} M \operatorname{det} F \\
& =\operatorname{det} M / \operatorname{det} A_{k k} .
\end{aligned}
$$

Finally, to show that $q(\phi(M)) \geqq q(M)$, we write the characteristic polynomial of $\phi(M)$ in the form

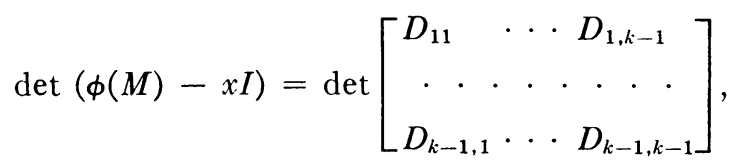

where $D_{i j}=\left(A_{i j}-\delta_{i j} x I\right)-A_{i k} A_{k k}^{-1} A_{k j}$. ( $\delta_{i j}$ is the Kronecker symbol.) Then clearly $\phi(M)-x I$ is the same as the matrix $\phi(Q(x))$, where $Q(x)$ agrees with the matrix $M$ except that the matrix $x I$ has been subtracted from each of the submatrices $A_{11}, A_{22}, \cdots, A_{k-1, k-1}$. (The construction of $\phi(Q(x))$ from $Q(x)$, and part (7) of Theorem 1, require only that $A_{\mathbf{k}}^{-1}$ exists, and not that $Q(x)$ be an $M$-matrix.) So as in (7), we have $\operatorname{det}(\phi(M)-x I)=\operatorname{det} \phi(Q(x))=\operatorname{det} Q(x) / \operatorname{det} A_{k k}$.

Now if $0<\lambda<q(M)$, Lemma 2 implies that $\operatorname{det} Q(\lambda)>0$. Also, $\operatorname{det} A_{k k}>0$, since it is a principal minor of $M$. Thus $\operatorname{det}(\phi(M)-\lambda I)$ $>0$, and so no real characteristic root of $\phi(M)$ is less than $q(M)$. I.e. $q(\phi(M)) \geqq q(M)$, completing our proof.

Applying Theorem 1, we obtain the following theorem about the Perron root of a nonnegative matrix.

ThEOREM 2. If $B$ is a nonnegative matrix of order $n=m k$, and $h>p(B)$, the Perron root of $B$, then

(9) $D=h I-\phi(h I-B)$ is a nonnegative matrix of order $m(k-1)$,

(10) $D_{i j} \geqq B_{i j}$ for $i, j=1,2, \cdots, m(k-1)$, and

(11) $p(B) \geqq p(D)$.

Proof. Let $M=h I-B$. Then $D_{i j}=h \delta_{i j}-\phi(M)_{i j} \geqq h \delta_{i j}-M_{i j}=B_{i j}$ $\geqq 0$, verifying (9) and (10). Also $h-p(D)=q(\phi(M)) \geqq q(M)=h-p(B)$, so that $p(B) \geqq p(D)$.

As in [2], the use of known lower bounds for the Perron root of $D$ leads to bounds for $p(B)$. Similarly, Theorem 1 gives new bounds for $q(M)$, by applying known upper bounds for the minimal characteristic root of the $M$-matrix $\phi(M)$.

Added in proof. The author has since discovered the fact that the 
matrix $\phi(M)$ can also be constructed using the method in [2, Lemma $1]$.

\section{REFERENCES}

1. Alfred T. Brauer, $A$ new proof of theorems of Perron and Frobenius on non-negative matrices, Duke Math. J. 24 (1957), 367-378.

2. Douglas E. Crabtree, Applications of $M$-matrices to non-negative matrices, Duke Math J. 33 (1966), 197-208.

3. Ky Fan, Note on M-matrices, Quart. J. Math (2) 11 (1960), 43-49.

4. M. Fiedler and V. Ptak, On matrices with non-positive off-diagonal elements and positive principal minors, Czechoslovak Math. J. 12 (1962), 382-400.

5. F. R. Gantmacher and M. G. Krein, Oszillationsmatrizen, Oszillationskerne, und kleine Schwingungen mechanischer Systeme, translated by Alfred Stohr, AkademieVerlag, Berlin, 1960.

UNIVERSITy OF MASSAChUSETTS 